\title{
On moduli spaces of semiquasihomogeneous singularities
}

\author{
Gert-Martin Greuel \\ Universität Kaiserslautern \\ Fachbereich Mathematik \\ Erwin-Schrödinger-Straße \\ D - 6750 Kaiserslautern
}

\author{
Gerhard Pfister \\ Humboldt-Univerität zu Berlin \\ Fachbereich Mathematik \\ Unter den Linden 6 \\ D - 1086 Berlin
}

\section{Contents}

Introduction

1 Versal $\mu$-constant deformations and kernel of the Kodaira-Spencer map

2 Existence of a geometric quotient for fixed Hilbert function of the Tjurina algebra

3 The automorphism group of semi Brieskorn singularities $\quad 13$

4 Problems 16

$\begin{array}{ll}\text { References } & 17\end{array}$ 


\section{Introduction}

Let $A=\mathrm{C}\left[\left[x_{1}, \ldots, x_{n}\right]\right] /(f)$ be the complete local ring of a hypersurface singularity. $A$ is called semiquasihomogeneous with weights $w_{1}, \ldots, w_{n}$ if $f=f_{0}+f_{1}, f_{0}$ a quasihomogeneous polynomial defining an isolated singularity and $\operatorname{deg} f_{0}<\operatorname{deg} f_{1}$. We assume that $w_{1}, \ldots, w_{n}$ are positive integers and let deg always denote the weighted degree, i.e. $\operatorname{deg} X^{\alpha}=w_{1} \alpha_{1}+\cdots+w_{n} \alpha_{n}$ for a monomial $X^{\alpha}=X_{1}^{\alpha_{1}} \cdot \ldots \cdot X_{n}^{\alpha_{n}}$. For an arbitrary power series $f, \operatorname{deg} f$ denotes the smallest weighted degree of a monomial occurring in $f$. By definition, all monomials of a quasihomogeneous polynomial have the same degree. The singularity with local ring $A_{0}=\mathbf{C}\left[\left[x_{1}, \ldots, x_{n}\right]\right] /\left(f_{0}\right)$ is called the principal part of $A$. If the moduli stratum of $A_{0}$ has dimension 0 , i.e. the $\tau-$ constant stratum in the semiuniversal deformation of $A_{0}$ is a reduced point, then $A_{0}$ is uniquely determined by the weights. Let $H^{i}=H^{i}\left(\mathbf{C}\left[\left[x_{1}, \ldots, x_{n}\right]\right]\right)$ be the ideal generated by all quasihomogeneous polynomials of degree $\geq i w, w:=\min \left\{w_{1}, \ldots w_{n}\right\}$. This (weighted) degree-filtration defines a Hilbert-function $\underline{\tau}$ on the Tjurina algebra of $A$ by

$$
\tau_{i}(A):=\operatorname{dim}_{\mathbf{C}} \mathbf{C}\left[\left[x_{1}, \ldots, x_{n}\right]\right] /\left(f, \frac{\partial f}{\partial x_{1}}, \ldots, \frac{\partial f}{\partial x_{n}}, H^{i}\right)
$$

We call $f$ or $A$ a semi Brieskorn singularity if the principal part is of BrieskornPham type, i.e. $f_{0}=x_{1}^{m_{1}}+\ldots+x_{n}^{m_{n}}, \operatorname{gcd}\left(m_{i}, m_{j}\right)=1$ for $i \neq j$. Then $f_{0}$ is quasihomogeneous with weight $\underline{w}=\left(w_{1}, \ldots, w_{n}\right)$, where $w_{i}=m_{1} \cdot \ldots \cdot \hat{m}_{i} \cdot \ldots \cdot m_{n}$, and degree $d=m_{1} \cdot \ldots \cdot m_{n}$, the moduli stratum is zero-dimensional and hence $f_{0}$ is uniquely determined by its weights (cf. [LP]). We are mainly interested in the classification of such singularities with respect to contact equivalence, i.e. in isomorphism classes of the local algebra $A$. With respect to this equivalence relation we shall prove:

Theorem There exists a coarse moduli space $\mathcal{M}_{\underline{w}, \underline{\underline{\tau}}}$ for all semiquasihomogeneous singularities with fixed principal part $A_{0}$, weight $\underline{w}$ and Hilbert function $\underline{\tau}$. $\mathcal{M}_{\underline{w}, \underline{\tau}}$ is an algebraic variety, locally closed in a weighted projective space.

We follow the general method to construct such moduli spaces (cf. [LP], [GP 1]):

1. We prove that the versal $\mu$-constant deformation $\tilde{X}_{\mu} \rightarrow \underline{H}_{\mu}$ of $A_{0}$ contains already all isomorphism classes of semiquasihomogeneous singularities with principal part $A_{0}$. (If we take the quotient of $\underline{H}_{\mu}$ by a natural action of the group of $d$-th roots of unity we obtain already a coarse moduli space with respect to right equivalence.)

2. This family contains analytically trivial subfamilies. They are the integral manifolds of a Lie-algebra $V_{\mu}$, the kernel of the Kodaira-Spencer map of the family. We prove that two singularities are isomorphic iff they are in one integral manifold of $V_{\mu}$.

3. The integral manifolds of the (infinite dimensional) Lie-algebra $V_{\mu}$ can be identified with the orbits of a solvable algebraic group $G$. Now the results of [GP 2] 
can be applied. We prove that the stratification $\left\{\underline{H}_{\mu, \tau}\right\}$ of $\underline{H}_{\mu}$ by fixing the Hilbert function has the properties required in [GP 2], i.e. $\underline{H}_{\mu, \tau} \rightarrow \underline{H}_{\mu, \underline{\tau}} / G$ is a geometric quotient and a coarse moduli space of all semiquasihomogeneous singularities with weight $\underline{w}$, Hilbert function $\underline{\tau}$ and principal part $A_{0}$. 


\section{Versal $\mu$-constant deformations and kernel of the Kodaira-Spencer map}

In this part we recall some known facts about the versal $\mu$-constant deformation and the kernel of the Kodaira-Spencer map.

Let $f_{0}=x_{1}^{m_{1}}+\ldots+x_{n}^{m_{n}}, n \geq 2, m_{i} \geq 2$ and $\operatorname{gcd}\left(m_{i}, m_{j}\right)=1$ if $i \neq j$.

Let $w_{i}=m_{1} \cdot \ldots \cdot \hat{m}_{i} \cdot \ldots \cdot m_{n}, \quad i=1, \ldots, n$ and $d=m_{1} \cdot \ldots \cdot m_{n}$ then $f_{0}$ is a quasihomogeneous polynomial with weight $\underline{w}=\left(w_{1}, \ldots, w_{n}\right)$ of degree $d$. Let $A_{0}=\mathbf{C}[[x]] /\left(f_{0}\right), x=\left(x_{1}, \ldots, x_{n}\right)$ and consider the deformation functor $D e f_{A_{0} \rightarrow \mathbf{C}}$ which consists of isomorphism classes of deformations of the residue morphism $A_{0} \rightarrow$ C. Geometrically, an element of $D e f_{A_{0} \rightarrow \mathbf{C}}$ is represented by a "deformation with section" of the singularity defined by $f_{0}(\mathrm{cf}$. [Bu]). It is not difficult to see (cf. $[\mathrm{LP}])$ that $D e f_{A_{0} \rightarrow \mathbf{C}}(\mathbf{C}[\epsilon])=(x) /\left(f_{0}+(x)\left(\frac{\partial f_{0}}{\partial x_{1}}, \ldots, \frac{\partial f_{0}}{\partial x_{n}}\right)\right)$ where $(x)$ denotes the ideal generated by $x_{1}, \ldots, x_{n}$. This vector space has a unique monomial base $\left\{x^{\alpha} \mid \alpha \in\right.$ $B\}, \alpha=\left(\alpha_{1}, \ldots, \alpha_{n}\right), x^{\alpha}=x^{\alpha_{1}} \cdot \ldots \cdot x^{\alpha_{n}}$ where $B=\left\{\alpha \in \mathbf{N}^{n} \backslash\{0\} \mid \alpha_{i} \leq m_{i}-2\right\} \cup$ $\left\{\left(0, \ldots, m_{i}-1,0, \ldots\right) \mid i=1, \ldots, n\right\}$ :

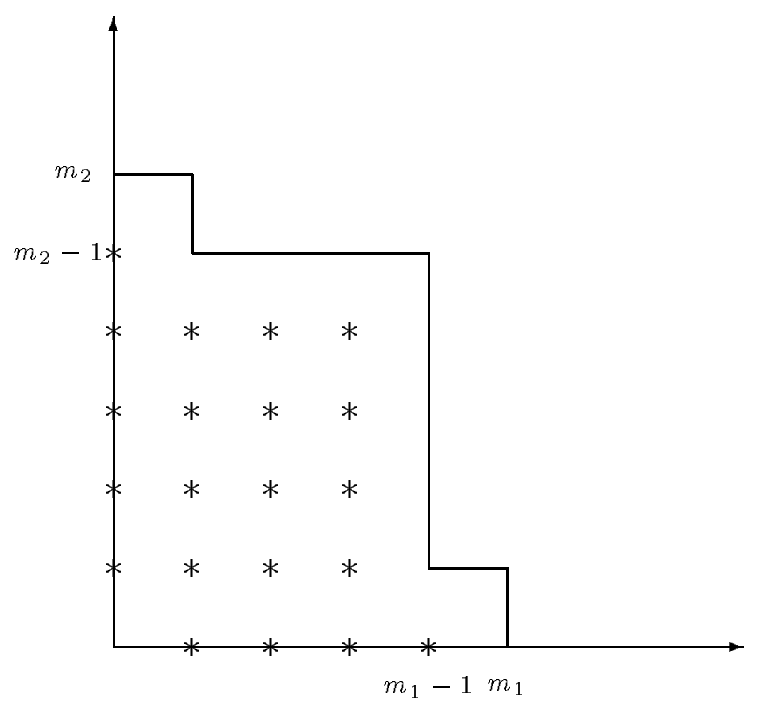

Figure 1: $B \quad(n=2)$

$D e f_{A_{0} \rightarrow \mathbf{C}}$ has a hull, the semiuniversal deformation, given on the ring level by $H \rightarrow H[[x]] / F$ with

$$
\begin{aligned}
F=F(T) & =f_{0}+\sum_{\alpha \in B} T_{d-|\alpha|} x^{\alpha} \\
H & =\mathrm{C}[T]
\end{aligned}
$$


$T=\left(T_{d-|\alpha|}\right)_{\alpha \in B}$ and $|\alpha|=\sum_{i=1}^{n} w_{i} \alpha_{i}$ which is by definition the degree of $x^{\alpha}$.

Notice that $F$ is quasihomogeneous if we define $\operatorname{deg} T_{i}=i$. We put $\underline{H}:=$ Spec $H \cong$ $\mathrm{C}^{N}, N=\# B=\prod_{i=1}^{n}\left(m_{i}-1\right)+n-1$, the base space of the semiuniversal deformation.

The moduli stratum, i.e. the $\tau$-constant stratum, is the zero point in $\underline{H}$.

Let $D e f_{A_{0} \rightarrow \mathbf{C}, \mathbf{C}^{*}}$ denote the functor of $\mathbf{C}^{*}$-equivariant deformations of $A_{0} \rightarrow \mathbf{C}$ (cf. $[\mathrm{Pi}])$ and let $D e f_{A_{0} \rightarrow \mathbf{C}}^{\mu}=\operatorname{Im}\left(D e f_{A_{0} \rightarrow \mathbf{C}, \mathbf{C}^{*}} \rightarrow D e f_{A_{0} \rightarrow \mathbf{C}}\right) . \quad D e f_{A_{0} \rightarrow \mathbf{C}}^{\mu}$ gives the $\mu^{-}$ constant deformations over a reduced base space. The functor $D e f_{A_{0} \rightarrow \mathbf{C}, \mathbf{C}^{*}}$ has a hull, the semiuniversal $\mu$-constant deformation, given by

$$
\begin{gathered}
H_{\mu} \rightarrow H_{\mu}[[x]] /\left(F_{\mu}\right) \text { with } \\
F_{\mu}=F_{\mu}(T)=f_{0}+\sum_{\alpha \in B_{-}} T_{d-|\alpha|} x^{\alpha} \\
H_{\mu}=\mathrm{C}\left[\left\{T_{d-|\alpha|}\right\}_{\alpha \in B_{-}}\right]
\end{gathered}
$$

where $B_{-}=\{\alpha \in B, d-|\alpha|<0\}$ :

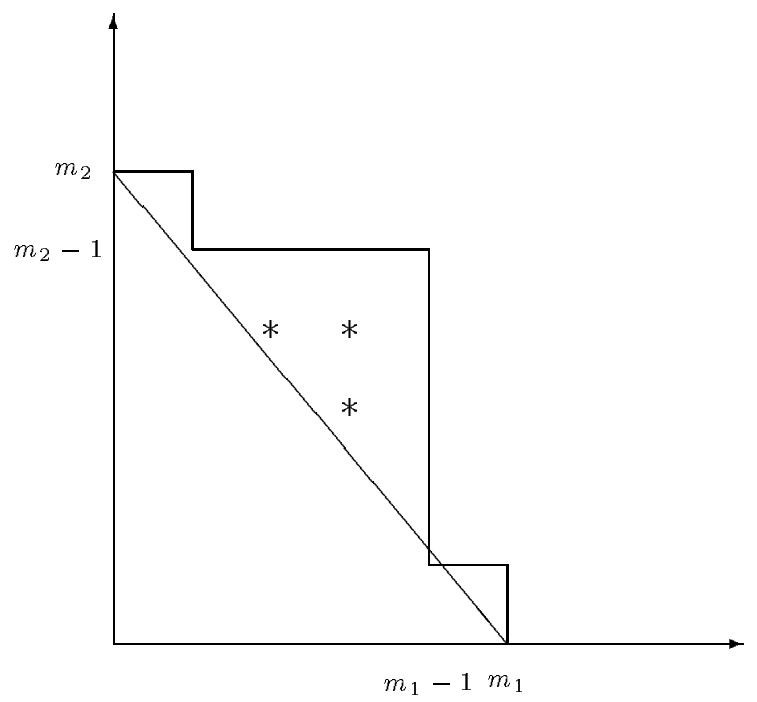

Figure 2: $B_{-} \quad(n=2)$

Remark 1.1 (1) The assumption $g c d\left(m_{i}, m_{j}\right)=1$ implies that except on the axes there are no extra integral points on the hyperplane $|\alpha|-d=0$, i.e. $f_{0}$ has no moduli. Moreover, it follows that on each hyperplane $|\alpha|=d^{\prime}, \alpha \in B$, there is at most one monomial $x^{\alpha}$, hence the elements of $B$ can be numbered by degree which turns out 
to be very convenient.

(2) For any $t \in \underline{H}_{\mu}:=\operatorname{Spec} H_{\mu}$ we have that $F_{\mu}(t)=f_{0}+f_{1} \in \mathbf{C}[[x]]$ is semiquasihomogeneous, with principal part $f_{0}$. The natural $\mathbf{C}^{*}$-actions, $c \circ x=\left(\ldots, c^{w_{i}} x_{i}, \ldots\right)$ and $c \circ t=\left(\ldots, c^{j} t_{j}, \ldots\right), c=\mathbf{C}^{*}$, have the property $F_{\mu}(c \circ t)(c \circ x)=c^{d} F_{\mu}(t)(x)$, in particular, $F_{\mu}(c \circ t)$ and $F_{\mu}(t)$ are right equivalent if $c^{d}=1$.

(3) The action of $\mu_{d}$ on $\underline{H}_{\mu}-\{0\}$ is faithful since $\mu_{d}$ acts with degree 0 and the $T_{i}$ have different degrees. This implies: if $\mathcal{X} \rightarrow S$ is any $\mu$-constant deformation of $A=\mathbf{C}[[x]] /\left(f_{0}+f_{1}\right)$, then there is an open covering $\left\{\mathcal{U}_{i}\right\}$ of $S$ such that $\left.\mathcal{X}\right|_{\mathcal{U}_{i}}$ is obtained via some base change $\varphi_{i}: \mathcal{U}_{i} \rightarrow \underline{H}_{\mu}$. By the following proposition $\varphi_{i} \circ \varphi_{j}^{-1}$ is equal to the $\mathbf{C}^{*}$-action given by some $d$-th root of unity $c_{i j}$. Since $\mu_{d}$ acts faithfully $\left\{c_{i j}\right\}$ defines a 1-Čech cocycle of $\mu_{d}$ on $S$. Hence, if $H^{1}(S, \mathbf{Z} / d \mathbf{Z})=0$, the $\varphi_{i}$ can be glued such that $\mathcal{X} \rightarrow S$ is globally obtained by some base change $S \rightarrow \underline{H}_{\mu}$.

Proposition 1.2 1. For any semiquasihomogeneous polynomial $f=f_{0}+f_{1}$ with principal part $f_{0}$ there is an automorphism $\varphi \in A u t \mathbf{C}[[x]]$ and $t \in \underline{H}_{\mu}$ such that $\varphi(f)=F_{\mu}(t)$.

2. If $F_{\mu}(t)$ and $F_{\mu}\left(t^{\prime}\right)$ are right equivalent for $t, t^{\prime} \in \underline{H}_{\mu}$ then there is a $d$-th root of unity $c$, such that $c \circ t=t^{\prime}$.

Corollary 1.3 Let $\mu_{d}$ denote the group of $d$-th roots of unity acting on $\underline{H}_{\mu}$ as above, then $\underline{H}_{\mu} / \mu_{d}$ is a coarse moduli space for semiquasihomogeneous polynomials $f$ with principal part $f_{0}$ and with respect to right equivalence.

For the notion of (coarse) moduli spaces see $[\mathrm{MF}]$ and $[\mathrm{Ne}]$. The fact that 1.3 is a corollary of 1.2 follows from general principals (cf. [Ne]; the assumption made there that all spaces are reduced is not necessary). See also remark 3.5.

Proof of 1.2: (1) is proved in [AGV], 12.6, theorem (p. 209).

(2) First notice that roots of unity cannot be avoided: take $f=x^{5}+y^{11}+x y^{9}, c_{1}^{5}=$ $c_{2}^{11}=1$ and $c=c_{1} c_{2}$. The automorphism $x \mapsto c^{11} x, y \mapsto c^{5} y$ maps $f$ to $x^{5}+y^{11}+c^{56} x y^{9}$.

The statement of (2) will follow from the following two lemmas:

Lemma 1.4 Let $f, g$ be semiquasihomogeneous with principal part $f_{0}$ as above and $\varphi \in$ Aut $\mathbf{C}[[x]]$ such that $\varphi(f)=g$. Then there is a $d$-th root of unity c such that

$$
\varphi\left(x_{i}\right)=c^{w_{i}} x_{i}+h_{i}, \operatorname{deg} h_{i}>w_{i} .
$$

Proof: Let $w_{1}<\ldots<w_{n}$. By proposition 3.2 we have $\operatorname{deg} \varphi \geq 0$, hence

$$
\varphi\left(x_{i}\right)=\sum_{j \geq i} c_{i j} x_{j}+\text { higher order terms. }
$$


Since $\varphi$ is an automorphism, $\prod_{i} c_{i i} \neq 0$, and $\varphi\left(x_{i}\right)=c_{i i} x_{i}+h_{i}, \operatorname{deg} h_{i}>w_{i}$. From $x_{1}^{m_{1}}+\ldots+x_{n}^{m_{n}}=c_{11}^{m_{1}} x_{1}^{m_{1}}+\ldots+c_{n n}^{m_{n}} x_{n}^{m_{n}}$ we deduce $c_{i i}^{m_{i}}=1$ and putting $c=\prod_{i} c_{i i}$ we obtain the result.

Lemma 1.5 Let $\varphi \in$ Aut $\mathbf{C}[[x]], \operatorname{deg} \varphi>0$, and $t, t^{\prime} \in \underline{H}_{\mu}$ such that $\varphi\left(F_{\mu}(t)\right)=$ $F_{\mu}\left(t^{\prime}\right)$. Then $t=t^{\prime}$.

Proof: By lemma 1.4, $\varphi\left(x_{i}\right)=x_{i}+h_{i}$. Hence $\varphi_{s}\left(x_{i}\right):=x_{i}+s h_{i}$ is a family of automorphisms of positive degree which connects $\varphi$ with the identity. Then $\varphi_{s}\left(F_{\mu}(t)\right)$ is a $\mathrm{C}^{*}$-equivariant family of isolated singularities, joining $F_{\mu}(t)$ and $F_{\mu}\left(t^{\prime}\right)$. This family may not be contained in $\underline{H}_{\mu}$ but it can be induced from $\underline{H}_{\mu}$ by a suitable base change (remark 1.1). But since $\underline{H}_{\mu}$ is everywhere miniversal and does, therefore, not contain trivial subfamilies with respect to right equivalence, $t=t^{\prime}$ as desired.

The Kodaira-Spencer map (cf. [LP]) of the functor $D e f_{A_{0} \rightarrow \mathbf{C}, \mathbf{C}^{*}}$ and of the family $H_{\mu} \rightarrow H_{\mu}[[x]] / F_{\mu}$

$$
\rho: \operatorname{Der}_{\mathbf{C}} H_{\mu} \longrightarrow(x) H_{\mu}[[x]] /\left(F_{\mu}+(x)\left(\frac{\partial F_{\mu}}{\partial x_{1}}, \ldots, \frac{\partial F_{\mu}}{\partial x_{n}}\right)\right),
$$

is defined by $\rho(\delta)=\operatorname{class}\left(\delta F_{\mu}\right)=\operatorname{class}\left(\sum_{\alpha \in B_{-}} \delta\left(T_{d-|\alpha|}\right) x^{\alpha}\right)$.

Let $\mathbf{V}_{\mu}$ be the kernel of $\rho . \mathbf{V}_{\mu}$ is a Lie-algebra and along the integral manifolds of $\mathbf{V}_{\mu}$ the family is analytically trivial (cf. [LP]).

In our situation it is possible to give generators of $\mathbf{V}_{\mu}$ as $H_{\mu}$-module:

Let $I_{\mu}=(x) H_{\mu}[[x]] /(x)\left(\frac{\partial F_{\mu}}{\partial x_{1}}, \ldots, \frac{\partial F_{\mu}}{\partial x_{n}}\right)$, then $I_{\mu}$ is a free $H_{\mu}-$ module and $\left\{x^{\alpha}\right\}_{\alpha \in B}$ is a free basis.

Multiplication by $F_{\mu}$ defines an endomorphism of $I_{\mu}$ and $F_{\mu} I_{\mu} \subseteq \oplus_{\alpha \in B_{-}} x^{\alpha} H_{\mu}$.

Especially, for $\alpha \in B$, define $h_{i, j}$ by

$$
x^{\alpha} F_{\mu}=\sum_{\beta \in B_{-}} h_{|\alpha|, d-|\beta|} x^{\beta} \text { in } I_{\mu} .
$$

Then $h_{i j}$ is homogeneous of degree $i+j$. This implies $h_{i j}=0$ if $i+j \geq 0$, in particular $h_{i j}=0$ if $i \geq(n-1) d-2 \sum w_{i}$. For $\alpha \in B$ and $|\alpha|<(n-1) d-2 \sum w_{i}$ let $\delta_{|\alpha|}:=\sum_{\beta \in B_{-}} h_{|\alpha|, d-|\beta|} \frac{\partial}{\partial T_{d-|\beta|}}$.

Proposition 1.6 (cf. [LP], proposition 4.5):

1. $\delta_{|\alpha|}$ is homogeneous of degree $|\alpha|$.

2. $\mathbf{V}_{\mu}=\sum_{\alpha} H_{\mu} \delta_{|\alpha|}$. 
Now there is a non-degenerate pairing on $I_{\mu}$ (the residue pairing) which is defined in our situation by $\langle h, k\rangle=h e s s(h \cdot k)$. Here for $h=\sum_{\alpha \in B} h_{\alpha} x^{\alpha} \in I_{\mu}$, hess $(h)=$ $h_{\left(m_{1}-2, \ldots, m_{n}-2\right)}$ which is the coefficient belonging to the Hessian of $f$.

Let the numbering of the elements of $B_{-}=\left\{\alpha_{1}, \ldots, \alpha_{k}\right\}$, be such that $\left|\alpha_{1}\right|<$ $\ldots<\left|\alpha_{k}\right|$ and denote by $\beta_{i}=\alpha_{k-i+1}^{\vee}, i=1, \ldots, k$, the dual exponents induced by the pairing, i.e. if $\gamma=\left(\gamma_{1}, \ldots, \gamma_{n}\right)$ then $\gamma^{\vee}=\left(m_{1}-2-\gamma_{1}, \ldots, m_{n}-2-\gamma_{n}\right)$.

Using the pairing one can prove the following

Proposition 1.7 There are homogeneous elements $m_{1}, \ldots, m_{k} \in H_{\mu}[[x]]$ with the following properties:

1. $\operatorname{deg} m_{i}=\left|\beta_{i}\right|$

2. If $m_{i} F_{\mu}=\sum_{j=1}^{k} \tilde{h}_{i j} x^{\alpha_{j}}$ in $I_{\mu}$ then $\tilde{h}_{i j}=\tilde{h}_{k-j+1, k-i+1}$

3. If $\tilde{\delta}_{\left|\beta_{i}\right|}:=\sum_{j=1}^{k} \tilde{h}_{i j} \frac{\partial}{\partial T_{d-\left|\alpha_{j}\right|}}$ then $\tilde{\delta}_{\left|\beta_{i}\right|}$ is homogeneous of degree $\left|\beta_{i}\right|$ and $\mathbf{V}_{\mu}=$ $\sum_{i=1}^{k} H_{\mu} \tilde{\delta}_{\left|\beta_{i}\right|}$.

In [LP] (proposition 5.6) this proposition is proved for $n=2$. The proof can easily be extended to arbitrary $n$. The important fact is the symmetry, expressed in 2 .

Let $L$ be the Lie-algebra generated (as Lie-algebra) by $\left\{\tilde{\delta}_{\left|\beta_{1}\right|}, \ldots, \tilde{\delta}_{\left|\beta_{k}\right|}\right\}$. Then $L$ is finite dimensional and solvable. $L_{0}:=[L, L]$ is nilpotent and $L / L_{0}=\mathbf{C} \tilde{\delta}_{\left|\beta_{1}\right|}$, where $\tilde{\delta}_{\left|\beta_{1}\right|}=\sum_{i=1}^{k}\left(\left|\alpha_{i}\right|-d\right) T_{d-\left|\alpha_{i}\right|} \frac{\partial}{\partial T_{d-\left|\alpha_{i}\right|}}$ is the Euler vector field (cf. [LP]).

Corollary 1.8 The integral manifolds of $\mathbf{V}_{\mu}$ coincide with the orbits of the algebraic group $\exp (L)$.

Now consider the matrix $M(T):=\left(\tilde{\delta}_{\left|\beta_{i}\right|}\left(T_{d-\left|\alpha_{j}\right|} \mid\right)\right)_{i, j=1, \ldots, k}=\left(\tilde{h}_{i j}\right)_{i, j=1, \ldots, k}$. Evaluating this matrix at $t \in \underline{H}_{\mu}$ we have

$$
\begin{aligned}
\operatorname{rank} M(t)= & \text { dimension of a maximal integral manifold of } \\
& \mathbf{V}_{\mu}(\text { resp. of the orbit of } \exp (\mathrm{L})) \text { at } t \\
= & \mu-\tau(t),
\end{aligned}
$$

where $\tau(t)$ denotes the Tjurina number of the singularity defined by $t$ i.e. of $F(x, t)$. 


\section{Existence of a geometric quotient for fixed Hilbert function of the Tjurina algebra}

We want to apply theorem 4.7 from [GP 2] to the action of $L_{0}$ on $\underline{H}_{\mu}$.

Theorem 2.1 ([GP 2]) Let $A$ be a noetherian $\mathbf{C}-$ algebra and $L_{0} \subseteq \operatorname{Der}_{\mathbf{C}}^{n i l} A$ a finite dimensional nilpotent Lie algebra. Suppose A has a filtration

$$
F^{\bullet}: 0=F^{-1}(A) \subset F^{0}(A) \subset F^{1}(A) \subset \ldots
$$

by subvector spaces $F^{i}(A)$ such that

$$
\delta F^{i}(A) \subseteq F^{i-1}(A) \text { for all } i \in \mathbf{Z}, \delta \in L_{0}
$$

Suppose moreover, $L_{0}$ has a filtration

$$
Z_{\bullet}: L_{0}=Z_{0}\left(L_{0}\right) \supseteq Z_{1}\left(L_{0}\right) \supseteq \ldots \supseteq Z_{e}\left(L_{0}\right) \supseteq Z_{e+1}\left(L_{0}\right)=0
$$

by sub Lie algebras $Z_{j}\left(L_{0}\right)$ such that

$$
\left[L_{0}, Z_{j}\left(L_{0}\right)\right] \subseteq Z_{j+1}\left(L_{0}\right) \text { for all } j \in \mathbf{Z}
$$

Let $d: A \rightarrow \operatorname{Hom}_{\mathbf{C}}\left(L_{0}, A\right)$ be the differential defined by $d(a)(\delta)=\delta(a)$ and let Spec $A=\cup U_{\alpha}$ be the flattening stratification of the modules

$$
\operatorname{Hom}_{\mathbf{C}}\left(L_{0}, A\right) / A d\left(F^{i}(A)\right) \quad i=1,2, \ldots
$$

and

$$
\operatorname{Hom}_{\mathbf{C}}\left(Z_{j}\left(L_{0}\right), A\right) / \pi_{j}(A(d A)) \quad j=1, \ldots, e,
$$

where $\pi_{j}$ denotes the projection $\operatorname{Hom}_{\mathbf{C}}\left(L_{0}, A\right) \rightarrow \operatorname{Hom}_{\mathbf{C}}\left(Z_{j}\left(L_{0}\right), A\right)$.

Then $U_{\alpha}$ is invariant under the action of $L_{0}$ and $U_{\alpha} \rightarrow U_{\alpha} / L_{0}$ is a geometric quotient which is a principal fibre bundle with fibre $\exp \left(L_{0}\right)$.

To apply the theorem we have to construct these filtrations and interpret the corresponding stratification in terms of the Hilbert function of the Tjurina algebra.

There are natural filtrations $H^{\bullet}(\mathbf{C}[[x]])$ resp. $F^{\bullet}\left(H_{\mu}\right)$ on $\mathbf{C}[[x]]$ resp. $H_{\mu}$ defined as follows:

Let $F^{i}\left(H_{\mu}\right) \subseteq H_{\mu}$ be the $\mathbf{C}$-vectorspace generated by all quasihomogeneous polynomials of degree $>-(i+1) w$ and $H^{i}(\mathbf{C}[[x]])$ be the ideal generated by all quasihomogeneous polynomials of degree $\geq i w$, where

$$
w:=\min \left\{w_{1}, \ldots, w_{n}\right\}
$$


For $t \in \underline{H}_{\mu}$ the Hilbert function of the Tjurina algebra

$$
\mathrm{C}[[x]] /\left(F_{\mu}(t), \frac{\partial F_{\mu}(t)}{\partial x_{1}}, \ldots, \frac{\partial F_{\mu}(t)}{\partial x_{n}}\right)
$$

corresponding to the singularity defined by $t$ with respect to $H^{\bullet}$ is by definition the function,

$$
n \mapsto \tau_{n}(t):=\operatorname{dim}_{\mathbf{C}} \mathbf{C}[[x]] /\left(F_{\mu}(t), \frac{\partial F_{\mu}(t)}{\partial x_{1}}, \ldots, \frac{\partial F_{\mu}(t)}{\partial x_{n}}, H^{n}\right)
$$

Notice that $\tau_{n}(t)=\tau(t)$ if $n$ is large and $\tau_{n}(t)$ does not depend on $t$ for small $n$. On the other hand, $\mu_{n}:=\mu_{n}(t):=\operatorname{dim}_{\mathbf{C}} \mathbf{C}[[x]] /\left(\frac{\partial F_{\mu}(t)}{\partial x_{1}}, \ldots, \frac{\partial F_{\mu}(t)}{\partial x_{n}}, H^{n}\right)$ does not depend on $t \in H_{\mu}$ and

$$
\mu_{n}-\tau_{n}(t)=\operatorname{rank}\left(\tilde{\delta}_{\left|\beta_{i}\right|}\left(T_{d-\left|\alpha_{j}\right|}\right)(t)\right)_{\left|\alpha_{j}\right|<n w} .
$$

This is an immediate consequence of the following fact:

Let

$$
T^{n}:=H_{\mu}[[x]] /\left(F_{\mu}, \frac{\partial F_{\mu}}{\partial x_{1}}, \ldots, \frac{\partial F_{\mu}}{\partial x_{n}}, H^{n}\right)
$$

then the following sequence is exact and splits:

$$
\begin{aligned}
& 0 \rightarrow \bigoplus_{\substack{\alpha \in B \\
|\alpha| \leq d}} H_{\mu} x^{\alpha} \rightarrow T^{\frac{d}{w}+i} \quad \rightarrow \quad \operatorname{Der}_{\mathbf{C}} H_{\mu} /\left(\mathbf{V}_{\mu}+\sum_{|\beta| \geq d+i w} H_{\mu} \frac{\partial}{\partial T_{d-|\beta|}}\right) \rightarrow 0 \\
& x^{\alpha} \mapsto \operatorname{class}\left(x^{\alpha}\right) \\
& \operatorname{class}\left(x^{\beta}\right) \mapsto \operatorname{class}\left(\frac{\partial}{\partial T_{d-|\beta|}}\right)
\end{aligned}
$$

and with the identification $\sum_{|\beta|<d+i w} H_{\mu} \frac{\partial}{\partial T_{d-|\beta|}} \simeq H_{\mu}^{N_{i}}$ we get $\operatorname{Der}_{\mathbf{C}} H_{\mu} /\left(\mathbf{V}_{\mu}+\sum_{|\beta| \geq d+i w} H_{\mu} \frac{\partial}{\partial T_{d-|\beta|}}\right) \simeq H_{\mu}^{N_{i}} / M_{i}$, where $M_{i}$ is the $H_{\mu}$-submodule generated by the rows of the matrix $\left(\tilde{\delta}_{\left|\beta_{\ell}\right|}\left(T_{d-\left|\alpha_{j}\right|}\right)\right)_{\left|\alpha_{j}\right|<d+i w}$.

The filtration $F^{\bullet}\left(H_{\mu}\right)$ has the property (F) because every homogeneous vector field of $L_{0}$ is of degree $\geq w$ (since $L / L_{0}$ is the Euler vector field, cf. $\S 1$ ) and $H_{\mu} d H_{\mu}=H_{\mu} d F^{s} H_{\mu}, s=\left[\frac{(n-1) d-2 \sum w_{i}}{w}\right]$ (since $n d-2 \sum w_{i}$ is the degree of the Hessian of $f$ and $T_{d-\left(n d-2 \sum w_{i}\right)}$ is the variable of smallest degree).

To define $Z_{i}\left(L_{0}\right)$ we use the duality defined in chapter 1 :

$$
\alpha \mapsto \alpha^{\vee}=\left(m_{1}-2-\alpha_{1}, \ldots, m_{n}-2-\alpha_{n}\right),
$$

and set $Z_{i}\left(L_{0}\right):=$ the Lie algebra generated by

$$
\left\{\tilde{\delta}_{|\alpha|} \in L_{0} \mid T_{d-\left|\alpha^{v}\right|} \in F^{s-i}\right\}
$$


$Z$. $\left(L_{0}\right)$ has the property $(\mathbf{Z})$ (for the definition of $\tilde{\delta}_{|\alpha|}$, see proposition 1.3 ). We have $F \in H^{n}$, hence $\mu_{n}=\tau_{n}$, if $n \leq \frac{d}{w}$ and $H^{n} \subset\left(\frac{\partial F_{\mu}}{\partial x_{1}}, \ldots, \frac{\partial F_{\mu}}{\partial x_{n}}\right)$ hence $\mu_{n}-\tau_{n}(t)$ is independent of $n$ if $n \geq \frac{d}{w}+s+1, s=\left[\frac{(n-1) d-2 \Sigma w_{i}}{w}\right]$ and equal to $\mu-\tau(t)$.

Therefore, we have $s+1$ relevant values for $\tau_{i}$, and we denote

$$
\begin{aligned}
\underline{\tau}(t) & :=\left(\tau_{\frac{d}{w}+1}(t), \ldots, \tau_{\frac{d}{w}+s+1}(t)\right), \\
\underline{\mu} & :=\left(\mu_{\frac{d}{w}+1}, \ldots, \mu_{\frac{d}{w}+s+1}\right) .
\end{aligned}
$$

Moreover, let $S=\left\{\underline{r}:=\left(r_{1}, \ldots, r_{s+1}\right) \mid \exists t \in \underline{H}_{\mu}\right.$ s.t. $\left.\underline{\mu}-\underline{\tau}(t)=\underline{r}\right\}$ and $\underline{H}_{\mu}=$ $\cup_{\underline{r} \in S} U_{\underline{r}}$ be the flattening stratification of the modules $T^{\frac{d}{w}+1}, \ldots, T^{w+1}$ i.e. $\left\{U_{\underline{r}}\right\}$ is the stratification of $\underline{H}_{\mu}$ defined by fixing the Hilbert function $\underline{\tau}=\underline{\mu}-\underline{r}$ with the scheme structure defined by the flattening property. We obtain:

Lemma 2.2 1. $(0, \ldots, 0,1)$ and $(0, \ldots, 0) \in S . U_{(0, \ldots, 0)}=\{0\}$ is a smooth point and $U_{(0, \ldots, 1)}$ is defined by $T_{d-|\beta|}=0$ for $|\beta|<n d-2 \sum w_{i}$ and $T_{2} \sum w_{i}-(u-1) d \neq 0$ (and hence is smooth).

2. Let $\bar{S}=S \backslash\{(0, \ldots, 0)\}$ and for $\underline{r} \in \bar{S}$ put

$$
\bar{U}_{\underline{r}}= \begin{cases}U_{\underline{r}} & \text { if } \underline{r} \neq(0, \ldots, 0,1) \\ U_{(0, \ldots 0,1)} \cup U_{(0, \ldots, 0)} & \text { if } \underline{r}=(0, \ldots, 0,1)\end{cases}
$$

Then

$\left\{\bar{U}_{\underline{r}}\right\}_{\underline{r} \in \bar{S}}$ is the flattening stratification of the modules $\left\{\mathrm{Hom}_{\mathbf{C}}\left(L_{0}, H_{\mu}\right) / H_{\mu} d F^{i} H_{\mu}\right\}$ and $\left.\overline{\{} \operatorname{Hom}_{\mathbf{C}}\left(Z_{i}\left(L_{0}\right), H_{\mu}\right) / \pi_{i}\left(H_{\mu} d F H_{\mu}\right)\right\}$.

As a corollary we obtain the following theorem (recall that $\mathbf{V}_{\mu}$ denotes the kernel of the Kodaira Spencer map, cf. $\S 1$ ):

Theorem 2.3 For $\underline{r} \in S, \bar{U}_{\underline{r}}$ is invariant under the action of $\mathbf{V}_{\mu}$ and $\bar{U}_{\underline{r}} \rightarrow \bar{U}_{\underline{r}} / \mathbf{V}_{\mu}$ is a geometric quotient. $\bar{U}_{\underline{r}} / \overline{\mathbf{V}}_{\mu}$ is locally closed in a weighted projective space.

Proof: Using the lemma and theorem 2.1 we obtain that $\bar{U}_{\underline{r}}$ is invariant under the action of $L_{0}$ and $\bar{U}_{\underline{r}} \rightarrow \bar{U}_{\underline{r}} / L_{0}$ is a geometric quotient. $L / L_{0}^{-}=\mathbf{C} \delta_{0}$ acts on $\bar{U}_{\underline{r}} / L_{0}$. By corollary 1.4, $\bar{U}_{\underline{r}} / V_{\mu}=\bar{U}_{\underline{r}} / L$. If $\underline{r} \neq(0, \ldots, 1)$, then $\bar{U}_{\underline{r}} / L_{0} \rightarrow \bar{U}_{\underline{r}} / L$ is a geometric quotient embedded in the corresponding weighted projective space. If $\underline{r}=(0, \ldots, 1)$ then $\bar{U}_{\underline{r}} / L_{0}=\bar{U}_{\underline{r}}$ and the geometric quotients $U_{(0, \ldots, 1)} \rightarrow U_{(0, \ldots, 1)} / \mathbf{V}_{\mu}, U_{(0, \ldots, 0)} \rightarrow$ $U_{(0, \ldots, 0)} / \mathbf{V}_{\mu}$ exist as smooth points.

It remains to prove the lemma.

Proof of lemma 2.2: Because of the exact sequence above the flattening stratification of the modules $\left\{T^{\frac{d}{w}+i}\right\}$ is also the flattening stratification 
of $\left\{\operatorname{Der}_{\mathbf{C}} H_{\mu} /\left(\mathbf{V}_{\mu}+\sum_{|\beta| \geq d+i w} H_{\mu} \frac{\partial}{\partial T_{d-|\beta|}}\right)\right\}$ resp. the flattening stratification of $\left\{H_{\mu}^{N_{i}} / M_{i}\right\}, M_{i}$ the submodule generated by the rows of the matrix $\left(\tilde{\delta}_{\left|\beta_{\ell}\right|}\left(T_{j}\right)\right)_{-i w<j}$. Now we have

$$
\tilde{\delta}_{|\beta|}\left(T_{d-|\alpha|}\right)=\tilde{\delta}_{\left|\alpha^{\vee}\right|}\left(T_{d-\left|\beta^{\vee}\right|}\right) .(*)
$$

By definition of $Z_{i}\left(L_{0}\right)$ we have

$$
H_{\mu} Z_{i}\left(L_{0}\right)=\sum_{T_{d-\mid \alpha \vee} \in F^{s-i}} H_{\mu} \tilde{\delta}_{|\alpha|}
$$

and with the identification

$$
\sum_{\beta \in B_{-}} H_{\mu} \frac{\partial}{\partial T_{d-|\beta|}}=H_{\mu}^{N}
$$

and $M^{i}$ the submodule generated by the rows of the matrix $\left(\tilde{\delta}_{|\alpha|}\left(T_{j}\right)\right)_{d+(s-i+1) w>\left|\alpha^{v}\right|}$ we obtain

$$
\operatorname{Der}_{\mathbf{C}} H_{\mu} / H_{\mu} Z_{i}\left(L_{0}\right) \cong H_{\mu}^{N} / M^{i}
$$

$(*)$ implies that the flattening stratification of the modules $\left\{T^{\frac{d}{w}+1}, \ldots, T^{s}\right\}$, which is $\underline{H}_{\mu}=\cup_{\underline{r} \in \bar{S}} \bar{U}_{\underline{r}}$, is the flattening stratification of the modules $\left\{\operatorname{Der}_{\mathbf{C}} H_{\mu} / H_{\mu} Z_{i}\left(L_{0}\right)\right\}_{i=1, \ldots, s}$.

On the other hand, the flattening stratification of the modules $\left\{H_{\mu}^{N} / M^{i}\right\}_{i=1, \ldots, s}$ is the flattening stratification of the modules

$$
\left\{\operatorname{Hom}_{\mathbf{C}}\left(Z_{i}\left(L_{0}\right), H_{\mu}\right) / \pi_{i}\left(H_{\mu} d H_{\mu}\right)\right\}
$$

because

$$
H_{\mu} Z_{i}\left(L_{0}\right)=\sum_{T_{d-\mid \alpha \vee} \in F^{s-i}} H \tilde{\delta}_{|\alpha|} .
$$

Furthermore the modules $\left\{\operatorname{Hom}_{\mathbf{C}}\left(L_{0}, H_{\mu}\right) / H_{\mu} d F^{i} H_{\mu}\right\}$ and $\left\{\operatorname{Der}_{\mathbf{C}} H_{\mu} / H_{\mu} L_{0}+\right.$ $\left.\sum_{|\beta| \geq d+i w} H_{\mu} \frac{\partial}{\partial T_{d-|\beta|}}\right\}$ have the same flattening stratification and they are flat on $U_{\underline{r}}$, because

$0 \rightarrow H_{\mu} \rightarrow \operatorname{Der}_{\mathbf{C}} H_{\mu} / H_{\mu} L_{0}+\sum_{|\beta| \geq d+i w} H_{\mu} \frac{\partial}{\partial T_{d-|\beta|}} \rightarrow \operatorname{Der}_{\mathbf{C}} H_{\mu} / \mathbf{V}_{\mu}+\sum_{|\beta| \geq d+i w} H_{\mu} \frac{\partial}{\partial T_{d-|\beta|}} \rightarrow 0$

is exact and splits on $\underline{H}_{\mu} \backslash\{0\}$.

This proves the lemma.

Remark 2.4 The main point of the lemma is that the flattening stratification of the modules $\left\{\operatorname{Hom}_{\mathbf{C}}\left(L_{0}, H_{\mu}\right) / H_{\mu} d F^{i} H_{\mu}\right\}$ is contained in the flattening stratification of the modules $\left\{\operatorname{Hom}_{\mathbf{C}}\left(Z_{j}\left(L_{0}\right), H_{\mu}\right) / \pi_{i}\left(H_{\mu} d H_{\mu}\right)\right\}$, hence is defined by the Hilbert function of the Tjurina algebra alone without any reference to the action of $L$. This is a consequence of the symmetry, expressed in proposition 1.3. 


\section{The automorphism group of semi Brieskorn singularities}

In this chapter we prove that the automorphism group of a semi Brieskorn singularity with principal part $f_{0}=x_{1}^{m_{1}}+\ldots+x_{n}^{m_{n}}, \operatorname{gcd}\left(m_{i}, m_{j}\right)=1$ for $i \neq j$, has no automorphisms of negative degree. A consequence of this result is that two points in $\underline{H}_{\mu}$ correspond to isomorphic singularities iff they are in one integral manifold of $\mathbf{V}_{\mu}$. Again, $d=m_{i} \cdot \ldots \cdot m_{n}$ denotes the degree of $f_{0}$.

Let $\mathbf{C}[[x]]_{m}$ denote the ideal of $\mathbf{C}[[x]]$ generated by power series of degree $\geq m$. An automorphism $\varphi$ of $\mathbf{C}[[x]]$ has degree $m$ if

$$
(\varphi-i d) \mathbf{C}[[x]]_{i} \subset \mathbf{C}[[x]]_{i+m}
$$

for any $i$. For $c \in \mathbf{C}^{*}$ let $\varphi_{c}: \mathbf{C}[[x]] \rightarrow \mathbf{C}[[x]]$,

$$
\varphi_{c}\left(x_{i}\right)=c^{w_{i}} x_{i}, i=1, \ldots, n
$$

denote the $\mathbf{C}^{*}$-action which is an automorphism of degree 0 .

Proposition 3.1 Let $f=f_{0}+\sum_{|\alpha|>d} a_{\alpha} x^{\alpha}, g=f_{0}+\sum_{|\alpha|>d} b_{\alpha} x^{\alpha}, \varphi \in$ Aut $\mathbf{C}[[x]]$ and $u \in \mathbf{C}[[x]]$ a unit such that $u f=\varphi(g)$. Then $\operatorname{deg} \varphi \geq 0$.

Remark 3.2 Let $\varphi \in$ Aut $\mathbf{C}[[x]]$ be of degree $\geq 0, f, g$ as above and $f=u \varphi(g)$ for some unit $u$. Then $\varphi\left(x_{i}\right)=c_{i} x_{i}+h_{i}$, $\operatorname{deg} h_{i}>w_{i}, u(0) c_{i}^{m_{i}}=1$. Putting $u_{i}$ some $m_{i}$-th root of $u(0)$ and $c=\prod_{i=1}^{m} u_{i} c_{i}$ we obtain $c^{d}=1, c^{w_{i}}=u_{i} c_{i}$, hence $u(0) \varphi(g)=\tilde{\varphi} \circ \varphi_{c}(g)$ and $f=\tilde{u} \tilde{\varphi} \circ \varphi_{c}(g)$ where $\operatorname{deg} \tilde{\varphi}>0$ and $\tilde{u}$ is a unit with $\tilde{u}(0)=1$.

Proof: We prove the proposition by induction on $n$, the case $n=1$ being trivial. We may assume that $m_{n}<\ldots<m_{1}$. Then we can write $\varphi\left(x_{1}\right)=\alpha_{1} x_{1}+h_{1}, \alpha_{1} \in \mathbf{C}$ and $\operatorname{deg} h_{1}>w_{1}=\min \left\{w_{1}, \ldots, w_{n}\right\}$.

First of all we shall see that $\alpha_{1} \neq 0$. Assume $\alpha_{1}=0$ then there is an $i>1$ such that $\varphi\left(x_{i}\right)=\beta x_{1}+h_{i}$ and $\operatorname{deg} h_{i}>w_{1}, \beta \neq 0$. Now

Using an automorphism of non-negative degree we may assume $\varphi\left(x_{i}\right)=x_{1}$.

$$
\left.u f\right|_{x_{1}=0}=g\left(\left.\varphi\left(x_{1}\right)\right|_{x_{1}=0}, \ldots,\left.\varphi\left(x_{i-1}\right)\right|_{x_{1}=0}, 0,\left.\varphi\left(x_{i+1}\right)\right|_{x_{1}=0}, \ldots\right)
$$

and

$$
\begin{gathered}
\left.\varphi\right|_{x_{1}=0}: \mathrm{C}\left[\left[x_{1}, \ldots, \hat{x}_{i}, \ldots, x_{n}\right]\right] \rightarrow \mathrm{C}\left[\left[x_{2}, \ldots, x_{n}\right]\right] \\
\left.x_{k} \mapsto \varphi\left(x_{k}\right)\right|_{x_{1}=0}
\end{gathered}
$$


is an isomorphism. Hence

$$
\left.\varphi\right|_{x_{1}=0}\left(g\left(x_{1}, \ldots, x_{i-1}, 0, x_{i-1}, \ldots, x_{n}\right)\right)=\left.u f\right|_{x_{1}=0} .
$$

But $g\left(x_{1}, \ldots, x_{i-1}, 0, x_{i+1}, \ldots, x_{n}\right)$ and $f\left(0, x_{2}, \ldots, x_{n}\right)$ define isolated singularities with different Milnor numbers (they are semiquasihomogeneous with weights $w_{1}, \ldots, \hat{w}_{i}, \ldots, w_{n}$ resp. $w_{2}, \ldots, w_{n}$ and degree $d$ ). This is a contradiction and implies $\alpha_{1} \neq 0$. Using $\varphi_{\alpha^{-1}}$ and an automorphism of positive degree we may assume now $\varphi\left(x_{1}\right)=x_{1}$.

Let us consider again the automorphism $\left.\varphi\right|_{x_{1}=0}$ of $\mathbf{C}\left[\left[x_{2}, \ldots, x_{n}\right]\right]$. Using the induction hypothesis we may assume $\left.\operatorname{deg} \varphi\right|_{x_{1}=0} \geq 0$. Since the inverse is also of nonnegative degree we may assume that $\left.\varphi\right|_{x_{1}=0}$ is the identity, i.e.

$$
\varphi\left(x_{1}\right)=x_{1} \text { and } \varphi\left(x_{i}\right)=x_{i}+x_{1} h_{i}, i=2, \ldots, n \text {. }
$$

Using again an automorphism of non-negative degree we may assume now that $h_{i}$ has only terms of degree $<w_{i}-w_{1}$. We have to prove that $h_{i}=0$.

If $h_{i}$ has only terms of degree $<w_{i}-w_{1}$ then $h_{i}$ does not depend on $x_{i}, \ldots, x_{n}$. We prove now that $h_{n}=0$.

We may assume that $g=x_{n}^{m_{n}}+x_{n}^{m_{n}-2} a_{2}+\ldots+a_{m_{n}}, a_{i} \in \mathbf{C}\left[\left[x_{1}, \ldots, x_{n-1}\right]\right]$. Indeed by the Weierstrass preparation theorem $g \cdot$ unit $=x_{n}^{m_{n}}+a_{1} x_{n}^{m_{n}-1}+\ldots$. This equality implies $\operatorname{deg} a_{1} x_{n}^{m_{n}-1}=\left(m_{n}-1\right) w_{n}+\operatorname{deg} a_{1}>d$ and consequently the automorphism defined by $x_{n} \rightarrow x_{n}-\frac{1}{m_{n}} a_{1}$ has positive degree. We may assume $a_{1}=0$ but this changes $\varphi\left(x_{n}\right)$ to $\varphi\left(x_{n}\right)=x_{n}+x_{1} h_{n}-\frac{1}{m_{n}} a_{1}$. Now $f \cdot u=\varphi\left(x_{n}^{m_{n}}+x_{n}^{m_{n}-2} a_{2}+\ldots\right)=$ $x_{n}^{m_{n}}+\left(m_{n} x_{1} h_{n}-a_{1}\right) x_{n}^{m_{n}-1}+\ldots$ and $\operatorname{deg} m_{n} x_{1} h_{n} x_{n}^{m_{n}-1}<d$. But this is only possible if $h_{n}=0$ because this term cannot be cancelled (the other $h_{i}$ do not depend on $x_{n}$ ). This implies $h_{n}=0$.

Now $\left.f \cdot u\right|_{x_{n=0}}=f\left(x_{1}, x_{2}+x_{1} h_{2}, \ldots, x_{n-1}+x_{1} h_{n-1}, 0\right)$ because the $h_{i}$ do not depend on $x_{n}$. Using again the induction hypothesis we obtain $h_{i}=0, i=2, \ldots, n-1$. This proves the proposition.

Corollary 3.3 If $t, t^{\prime} \in \underline{H}_{\mu}$ define isomorphic singularities then $t$ and $t^{\prime}$ are in the same maximal integral manifold of $\mathbf{V}_{\mu}$.

Proof: Let $F_{\mu}(t)=u \varphi\left(F_{\mu}\left(t^{\prime}\right)\right), u \in \mathbf{C}[[x]]$ a unit and $\varphi \in$ Aut $\mathbf{C}[[x]]$. By the proposition $\operatorname{deg} \varphi \geq 0$. Using remark 3.2 there is a $d^{\prime}$ th root of unity $c$ such that $F_{\mu}(x, t)=u \varphi\left(F_{\mu}\left(c^{-1} \circ x, t^{\prime}\right)\right)=u \varphi\left(F_{\mu}\left(x, c \circ t^{\prime}\right)\right)$ and such that $\operatorname{deg} \varphi>0$ and $u(0)=1$. Then

$$
G(z):=u\left(z^{w_{1}} x_{1}, \ldots, z^{w_{n}} x_{n}\right) \cdot F_{\mu}\left(\left(\frac{1}{z^{w_{1}}} \varphi\left(z^{w_{1}} x_{1}\right), \ldots, \frac{1}{z^{w_{n}}} \varphi\left(z^{w_{n}} x_{n}\right), c \circ t^{\prime}\right)\right.
$$


is an unfolding of $G(0)=F_{\mu}\left(x, c \circ t^{\prime}\right)$. This unfolding can be induced by the universal unfolding by remark 1.1, i.e. there exists a family of coordinate transformations $\underline{\psi}(z,-)$ and a path $v$ in $\underline{H}_{\mu}$ such that

$$
G(z)=F_{\mu}\left(\psi_{1}(z, x), \ldots, \psi_{n}(z, x), v(z)\right)
$$

and $v(0)=c \circ t^{\prime}, \psi_{i}(0, x)=x$. By $[\mathrm{AGV}]$ we may assume that $\underline{\psi}(z,-)$ has positive degree.

Because $F_{\mu}(x, t)=F_{\mu}(\psi(1, x), v(1))$ we obtain $v(1)=t$ by lemma 1.5. This implies that $t$ and $c \circ t^{\prime}$ are in an analytically trivial family, i.e. in an integral manifold of $\mathbf{V}_{\mu}$ which contains the $\mathbf{C}^{*}$-orbits (cf. $\S 1$ ). Hence the result.

This finishes the second step of the approach. Together with the theorem of chapter 2 we obtain the theorem stated in the introduction:

Theorem 3.4 There exists a coarse moduli space $\mathcal{M}_{\underline{w}, \underline{\tau}}=\bar{U}_{\underline{\mu-\underline{r}}} / \mathbf{V}_{\mu}$ of all semiquasihomogeneous hypersurface singularities $A=\mathbf{C}[[\underline{x}]] /(f)$ wit $\bar{h}$ fixed principal part $A_{0}=\mathbf{C}[[\underline{x}]] /\left(f_{0}\right)$, weight $\underline{w}$ and Hilbert function $\underline{\tau} . \mathcal{M}_{\underline{w}, \underline{\tau}}$ is an algebraic variety, locally closed in a weighted projective space.

Remark 3.5 To be more precise, first of all $\mathcal{M}_{\underline{w}, \underline{\tau}}$ is a coarse moduli space for the functor which associates to any complex space germ $S$ the set of isomorphism classes of flat families over $S$ of quasihomogeneous hypersurface singularities with fixed principal part $A_{0}$, weight $\underline{w}$ and Hilbert function $\tau$. The category of base spaces is that of germs since we constructed $\mathcal{M}_{\underline{w}, \underline{\tau}}$ from the versal family over $\underline{H}_{\mu}$ which has the versality property only for germs. But by remark 1.1(3) we can actually enlarge the category of base spaces to all complex spaces $S$ for which $H^{1}(S, \mathbf{Z} / d \mathbf{Z})=0$. The same applies to the coarse moduli space $\underline{H}_{\mu} / \mu_{d}$ for functions with respect to right equivalences (cf. corollary 1.3). 


\section{Problems}

We use the notations of chapter 1 .

4.1 In the case $n=2$ (plane curves) the following holds (cf. [LP]): let $\left\{S_{\tau}\right\}$ be the stratification of $\underline{H}_{\mu}$ by constant Tjurina number, then

(i) $S_{\tau} \neq \emptyset$ if $\tau_{\min } \leq \tau \leq \mu$ (i.e. all possible Tjurinia numbers occur).

(ii) $\operatorname{dim} S_{\tau} / \mathbf{V}_{\mu} \geq \operatorname{dim} S_{\tau^{\prime}} / \mathbf{V}_{\mu}$ if $\tau \leq \tau^{\prime}$ (i.e. the number of moduli decreases when $\tau$ becomes more special).

(iii) $S_{\tau_{m i n}} / \mathbf{V}_{\mu}$ is a quasismooth algebraic variety.

In $[\mathrm{LP}]$ is an example showing that (i) and (ii) are wrong in higher dimension.

Problem 1: Does (iii) hold in higher dimension?

Problem 2: Find the dimensions of $\underline{H}_{\mu} / \mathbf{V}_{\mu}$.

4.2 In chapter 3 we proved that for semi Brieskorn singularities with principal part $f_{0}=x_{1}^{m_{1}}+\ldots+x_{n}^{m_{n}}, \operatorname{gcd}\left(m_{i}, m_{j}\right)=1$ for $i \neq j$ the automorphisms have non-negative degree.

Problem 3: Is this true for all quasihomogeneous singularities with zerodimensional moduli stratum?

A solution of this problem would solve the moduli problem for this class of semiquasihomogeneous singularities. 


\section{References}

[AGV] Arnol'd, V.I.; Gusein-Zade, S.M.; Varchenko, A.N.: Singularities of Differentiable Maps, Vol. I, Boston-Basel-Stuttgart: Birkhäuser 1985.

[Bu] Buchweitz, A.: Thesis, Université Paris VII, (1981).

[GP 1] Greuel, G.-M.; Pfister, G.: Moduli for singularities. Preprint 207, Kaiserslautern, 1991. To appear in the proceedings of the Lille conference on singularities, 1991.

[GP 2] Greuel, G.-M.; Pfister, G.: Geometric quotients of unipotent group actions. To appear in Proc. Lond. Math. Soc.

[LP] Laudal, O.A.; Pfister, G.: Local moduli and singularities. Lecture Notes in Math., Vol. 1310. Berlin-Heidelberg-New York: Springer 1988.

[MF] Mumford, D.; Fogarty, J.: Geometric Invariant Theory. (Second, enlarged edition). Ergb. Math. Grenzgeb. Bd. 34. Berlin-Heidelberg-New York: Springer 1982 .

[Ne] Newstead, P.E: Introduction to Moduli Problems and Orbit Spaces. Tata Inst. Fund. Res. Lecture Notes 51. Berlin-Heidelberg-New York: Springer 1978 .

[Pi] Pinkham, H.C.: Normal surface singularities with $\mathrm{C}^{*}$ action. Math. Ann. 227, 183-193 (1977). 